\title{
Inheritance of Resistance to Celery mosaic virus in Celery
}

\author{
Vincent D'Antonio, Department of Vegetable Crops, Bryce Falk, Department of Plant Pathology, and Carlos F. \\ Quiros, Department of Vegetable Crops, University of California, Davis 95616
}

\begin{abstract}
D'Antonio, V., Falk, B., and Quiros, C. F. 2001. Inheritance of resistance to Celery mosaic virus in celery. Plant Dis. 85:1276-1277.
\end{abstract}

Screening of the Apium germ plasm collection for resistance to Celery mosaic virus (CeMV) revealed four resistant accessions: a celeriac, a feral celery line, and two wild celery species. The feral celery, which is genetically closest to cultivated celery, A. graveolens var. dulce, was used to generate backcross and F2 progenies to determine the inheritance of the resistance. Resistance was recessive and determined by a single locus named $\mathrm{cmv}$. The simple inheritance of this trait will allow the development of celery lines resistant to CeMV.

Additional keywords: Apium, disease resistance, Potyvirus

Celery mosaic virus (CeMV) is a problem to celery (Apium graveolens L. var. dulce (Mill.) Pers.) growers in much of California and other producing states. CeMV is transmitted by aphids in a nonpersistent manner (5-7). It is a member of the genus Potyvirus and is restricted in host range to the Apiaceae (5-7). CeMV has also been known previously as Western celery mosaic virus, Apium virus 1, and Celery ringspot virus $(2,4)$. Disease symptoms include the formation of mosaic patterns on leaves, veinclearing, mottling brown spotting, leaflet twisting or cupping, and stunting. Occasionally, this disease causes serious losses $(3,5)$. The main method of control for the disease caused by CeMV in California has been a celery-free period in areas where celery is grown commercially (3). The recommended length of the celery-free period is 2 to 3 months, which allows the life cycles of the aphid vectors and virus host plants to be broken and provides a host-free period for CeMV. The objective of this paper is to report sources of resistance to CeMV in celery and the inheritance of this resistance.

\section{MATERIALS AND METHODS}

Plant materials. The initial screening was done on 85 celery accessions from our collection. These included 43 celery, 22 celeriac, 15 smallage accessions, and accessions of 4 wild species (A. panul, A. chilense, A. prostratum, and A. nodiflorum). Fifteen 8-week-old plants of each

Corresponding author: C. F. Quiros

E-mail: cfquiros@ucdavis.edu

Accepted for publication 24 August 2001.

Publication no. D-2001-1022-03R

(C) 2001 The American Phytopathological Society line were screened. Screening was done using an isolate of CeMV collected by us from CeMV-infected celery in the Salinas Valley of California. This virus was subsequently propagated in the greenhouse by standard mechanical inoculation in celery (cv. Tall Utah). For screening accessions, three fresh symptomatic celery leaves from a greenhouse-grown CeMV-infected celery plant (cv. Tall Utah 50-72R) were homogenized using a mortar and pestle in 10 $\mathrm{ml}$ of phosphate buffer $(0.03 \mathrm{M}$ potassium phosphate, $0.1 \%$ sodium sulfite, $\mathrm{pH}$ 7.0). An abrasive at a concentration of approximately 1\% (Celite, Fisher, Los Angeles, CA) was added to the solution, and 8week-old plants to be screened were rubbed with the inoculum using a pestle (1). In the initial screening, plants were scored visually for symptoms of CeMV infection. In order to provide more definitive data, young leaves of all plants in the initial screening (plus healthy and CeMVinfected controls) were tested for CeMV by enzyme-linked immunosorbent assay (ELISA) to confirm the presence or absence of CeMV. Double-antibody sandwich ELISA was conducted as described previously (1) using polyclonal antiserum prepared to purify virions of the California CeMV (B. Falk, unpublished). Samples were tested as paired replicates. Absorbance values $\left(A_{405}\right)$ were assessed 30,60 , and $120 \mathrm{~min}$ after addition of substrate. Each plate contained samples from two healthy and two CeMV-infected Tall Utah plants.

Screening and genetic analysis. After the first screening, one selected resistant plant of accession A69, a feral accession of A. graveolens collected from Oso Flaco, CA, was used to cross to the Fusarium resistant celery cultivar Promise to obtain an F1 population. The resulting hybrid was selfed to obtain the F2. An F1 plant was backcrossed to both a resistant plant of accession A69 and the susceptible Promise. We tested for resistance a total of 345 plants. These included plants from both parental accessions, F1, F2, and both firstbackcross populations, as well as 15 plants of Tall Utah 52-75 as a CeMV-susceptible control. Plants were started from seed in growing trays. Greenhouse temperature was $22^{\circ} \mathrm{C}$ days and $18^{\circ} \mathrm{C}$ at night. Sixweek old seedlings from all genotypes described above were transplanted into 10$\mathrm{cm}$ pots, completely randomized, and mechanically inoculated (1) with CeMV 2 weeks later. The first readings (visual scorings) were taken 3 weeks postinoculation and then each week thereafter for a total of seven readings. Plants were visually scored from 1 to 5 , with 1 being free of symptoms and 5 being most severe, showing stunting and obvious mosaic in young leaves. Readings were always taken on the youngest leaves, as these were most consistently symptomatic.

Scoring was done as follows: $1=$ symptom free; $2=$ slight veinclearing or mosaic appearing on one leaf; $3=$ slight veinclearing and mosaic on two or more leaves; $4=$ pronounced mosaic and veinclearing on two or more leaves; and $5=$ most striking mosaic and veinclearing, stunting.

Because so many of the F2 plants did not show obvious symptoms, all asymptomatic F2 plants as well as four randomly selected F2 symptomatic plants were tested for CeMV by back-inoculation (using standard conditions of mechanical inoculation) to susceptible cv. Tall Utah plants. Randomly selected parental controls were tested also. Symptoms on back-inoculated plants were evaluated visually 4 weeks postinoculation. For analysis, any plant with a score of 2 or higher was considered susceptible. Only symptom-free plants were considered resistant. Goodness-of-fit $\chi^{2}$ test was done on the final reading.

\section{RESULTS}

The preliminary accession screening revealed that one celeriac (A31) and a feral A. graveolens accession (A69) had both CeMV-resistant and -susceptible plants. Additionally, all the plants from the two wild species, A. chilense (A73) and A. prostratum (A230), were resistant to CeMV. ELISA allowed us to unequivocally identify CeMV-infected plants. All symptomatic CeMV-infected plants, whether commercial celery types or wild species, gave strong ELISA reactions $(0.5-1.5$ $\left.A_{405}\right)$ well above those observed for healthy plants $\left(0.1-0.2 A_{405}\right)$. Plants scored as 
resistant to CeMV had ELISA reactions similar in intensity to those for healthy plants $\left(0.1-0.2 A_{405}\right)$. CeMV-infected plants of true celery types also showed obvious symptoms of infection, while symptoms were sometimes less obvious in wild species. Based on this result, the feral accession A69, which belonged to the same species as cultivated celery, was used for resistance inheritance studies.

Some of the original resistant plants of this accession were selfed in attempts to create a pure resistant line. Plants of this line were subsequently used as controls for studies on inheritance of resistance to CeMV. Preliminary screening of celery plants for CeMV resistance showed that not all the susceptible plants, especially those in the segregating populations, displayed symptoms at the same time (Fig. 1). Therefore, it was evident that for reliable visual scoring, the scoring should take place 8 to 9 weeks after inoculation. We found that at 8 weeks after inoculation, the number of new plants showing symptoms reached a plateau, so the scoring was ter- minated on the ninth week. At this time, the final count and data analysis were performed. As expected, all of the plants in the two susceptible controls showed symptoms, whereas all those of the resistant parent did not (Table 1). All but one plant of the F1 progeny displayed typical symptoms of CeMV infection, as did all plants of the first backcross to the susceptible parent Promise. The F2 progeny segregated in Mendelian fashion, fitting a 3 susceptible: 1 resistant ratio. The backcross of the $\mathrm{F} 1$ tible: 1 resistant ratio. Back-inoculation tests also confirmed that the symptomless plants, classified as resistant, were CeMV-free, since none of the plants inoculated with leaf material from these asymptomatic plants became infected with CeMV, while those inoculated with extracts of leaves from symptomatic plants did.

\section{DISCUSSION}

The work presented here shows identification of a source of resistance to CeMV in Apium spp. Resistant plants failed to show progeny to the resistant parent fit a 1 suscep-

symptoms of CeMV infection, and ELISA and back-inoculation to susceptible celery plants confirmed that the asymptomatic plants were CeMV-free. Furthermore, by genetic analysis using resistant and susceptible sources, we were able to determine the inheritance of resistance.

The inheritance analysis based on analyses of plants from the F1, both reciprocal backcrosses, and the F2 generation showed that the resistance to CeMV in celery was recessive and determined by a single locus, which we named $c m v$. These data fit the expected ratios for a recessive single gene (Table 1). It should be mentioned, however, that the $\mathrm{BC} 1$ progeny to the resistant parent was skewed, resulting in an excess of susceptible plants. However, this deviation was not large enough to reject the single gene hypothesis. We have now found two molecular markers associated to the $c m v$ locus that will be useful for marker-assisted selection (8). Interestingly, the susceptible controls showed symptoms early and uniformly after inoculation, whereas some of the plants of the segregating progenies took longer to develop symptoms. This may be related to the variable genetic background and morphology of the plants in the segregating populations. In these tests, plants were allowed to develop and readings were taken over several weeks until the number of plants showing symptoms leveled off ( 9 weeks postinoculation).

Given the simple mode of inheritance, it will be possible to breed CeMV-resistant celery by incorporating the $\mathrm{cmv}$ allele into Fusarium-resistant lines and standard varieties. This, along with celery-free periods, should give better and more effective control of CeMV in celery.

\section{ACKNOWLEDGMENTS}

We thank the California Celery Research Advisory Board for supporting this work.

\section{LITERATURE CITED}

1. Falk, B. W., and Purcifull, D. E. 1983. Development and application of an enzyme-linked immunosorbent assay (ELISA) test to index lettuce seeds for lettuce mosaic virus in Florida. Plant Dis. 67:413-416.

2. Hollings, M. 1964. Some properties of five viruses of celery (Apium graveolens L.) in Britain. J. Hortic. Sci. 39:130-141.

Fig. 1. Development of symptoms over time in F2 celery plants inoculated with Celery mosaic virus (CeMV). Plants were observed for typical symptoms of CeMV infection beginning 3 weeks after inoculation. At 8 weeks postinoculation, the number of plants showing symptoms reached a maximum.

Table 1. Number of plants segregating for Celery mosaic virus (CeMV) infection in F2 and BC progenies

\begin{tabular}{lcccc}
\hline Lines $^{\mathbf{y}}$ & Resistant & Susceptible & $\chi^{2}$ & $\boldsymbol{P}$ \\
\hline A69 & 25 & 0 & - & - \\
Promise & 0 & 15 & - & - \\
F1 & $1(0)^{\mathrm{z}}$ & $24(25)$ & & - \\
F2 & $22(25)$ & $78(75)$ & 0.48 & 0.45 \\
BC1a/(F1×A69) & $64(75)$ & $86(75)$ & 3.22 & 0.07 \\
BC1b/(F1×Promise) & 0 & 30 & - & - \\
\hline
\end{tabular}

${ }_{\mathrm{y}} \mathrm{A} 69$ and Promise are the parental resistant and susceptible lines, respectively. BC1a is first backcross to resistant A69 accession and $\mathrm{BC} 1 \mathrm{~b}$ is first backcross to susceptible Promise.

${ }^{\mathrm{z}}$ In parentheses, expected number of plants for single locus segregation.
3. Milbrath, D. G., and Ryan, H. J. 1938. A method of control of western celery mosaic. Bull. Dep. Agric., Calif. 27:290-295.

4. Pemberton, A. W., and Frost, R. R. 1986. Virus diseases of celery in England. Ann. Appl. Biol. 108:319-331.

5. Severin, H. H. P., and Freitag, J. H. 1938. Western celery mosaic. Hilgardia 11:493-558.

6. Shepard, J. F., and Grogan, R. G. 1967. Partial purification, properties, and serology of western celery mosaic virus. Phytopathology 57:1104-1110.

7. Simons, J. N., and Sylvester, E. S. 1953. Acquisition threshold periods of western celery mosaic virus for four species of aphids. Phytopathology 43:29-31.

8. Ruiz, J. J., Pico, B., Li, G., D'Antonio, V., Falk, B., and Quiros, C. F. 2001. Identification of markers linked to a Celery Mosaic Virus resistance gene in celery. J. Am. Soc. Hortic. Sci. 126:427-431. 